\title{
LES EFFETS DE LA REFRACTION ATMOSPHERIQUE SUR LES COORDONNEES TANGENTIELLES EN ASTROMETRIE
}

\author{
J. DOMMANGET
}

Observatoire Royal de Belgique, Belgium

Résumé. On montre que les coordonnées tangentielles considérées en astrométrie et affectées de la réfraction, peuvent toujours s'écrire, même pour des champs pouvant s'étendre théoriquement jusqu'à $90^{\circ}$, sous la forme:

$$
\begin{aligned}
& X_{r}=X+a X_{a}+R_{x} \\
& Y_{r}=Y+a Y_{a}+R_{y},
\end{aligned}
$$

où $a$ est le coefficient du premier terme de l'expression de la réfraction:

$$
r=a \operatorname{tg} \xi+b \operatorname{tg}^{3} \xi
$$

$X_{a}$ et $Y_{a}$, des expressions calculables aisément pour chaque point du cliché et $R_{x}$ et $R_{y}$, les restes des développements des coordonnées réfractées $X_{r}$ et $Y_{r}$. Ces restes sont fonctions de $a$ et de $b$ théoriquement, mais leur calcul pour chaque point du cliché peut se faire en toute rigueur même avec des valeurs approchées de ces coefficients. Introduits sous cette forme dans les formules de réduction des clichés, les effets de la réfraction atmosphérique n'apparaissent que par le seul paramètre inconnu $a$, et de manière linéaire. 